\title{
Biometric Palm Print Recognition using Spatial Classifiers and Morphological Texture Segmentation
}

\author{
A.Kanchana \\ Research Scholar, \\ Dept. of CSE, \\ Mahendra Engineering College for women,
}

\author{
S.Arumugam, PhD. \\ Principal, \\ Nandha College of Technology, \\ Erode
}

\begin{abstract}
Automatic human identification is one of the most penetrating tasks to meet growing demand for rigorous security. The usage of biometrics has been largely used in the identification and recognition of criminals and has become an essential tool for law and order enforcement departments. The biometrics- based automated human identification and recognition process is now become highly popular in wide range of civilian applications and has processed as a powerful substitute to traditional password or token identification systems. Human palms are easier to present and perform for imaging and can reveal a wide range of information.
\end{abstract}

Palm print recognition uses the person's palm as a biometric for identifying or verifying the individuals. The application includes deployment for access control at points of entrance like airports, federal buildings and in highly sensitive places. Our existing work used multiple correlation filters per class for performing palm print classification algorithm. Correlation filters are classified as two classes filters that produce sharp peak for known class and noisy output for unknown sample class using images from the PolyU database.

In this work Advanced and Fast Correlation Based Feature for Palm-print Recognition (AFCBF) is proposed based on modified Correlation Filter classifier with spatial entities to identify more line features of the palm print very efficiently and in a stochastic manner. Experimental assessment using a CASIA Palm print Image database has illustrated the efficient performance of AFCBF compared to the existing palm-print classification used correlation filter classifiers.

Keywords : Gabor Wavelet transform (GWT) , Hamming Distance (HD),Palm-print orientation code (POC), competitive code (CompCode).

\section{INTRODUCTION}

The increase in biometric based image matching technique has led to the development of various authentication systems. Biometric authentication has become one of the significant areas in research over the past decade with high demands in automated personal identification. A palm print can be defined as an of the palm region of the hand. It can refer to either an online image or offline image. The palm includes features like principal lines, wrinkles and ridges. Palm print recognition refers to a biometric model that predominantly uses the structure of a person's palm print for recognition or identification purposes.

Palm print patterns are reliable biometric forms. Palm print recognition needed minimum cooperation from user for palm print extraction. The features of palm print are palm prints are more discriminative than fingerprints and palm print recognition focused on defining and extracting features from palm print patterns. Amidst many biometric techniques, palm print recognition is one of the most decisive approaches, since a palm print, contains many features such as singular points, texture and principle lines.

A major approach for palm print recognition in today's scenario is to extract feature vectors that correspond to individual palm print images and from the images obtained we perform palm print matching based on some distance metrics like singular points, texture, principle lines, wrinkles or ridges. One of the problems faced in feature based palm print recognition is that the matching performance is characterized by many parameters in feature extraction process that may differ depending on environmental factors of palm print image acquisition.

Recent research efforts address the problem of palm print recognition in large databases, and achieve very low error rates. The existing palm print classification used correlation filter classifiers, using templates requires neither feature extraction stage nor defines a specific set of features. Preliminary results obtained for palm print recognition using correlation filter classifiers were very promising. My works presents plan to improve correlation filter classifier with spatial entities to identify more unique features of the palm print.

\section{LITERATURE REVIEW}

One of the most extravagantly developing and influential techniques for identification currently lies in the field of Biometrics. This paper [3] involves the usage of certain inherent traits of humans in order to identify them. Biometrics can be divided into two discrete types: Physiological and behavioral. One of the most accurate and reliable of the Physiological Biometrics Techniques is Palm print Identification. Palm Prints have been in use for over a century as identification standards.

The paper [1] uses two novel approaches to improve the overall results and performance of palm-vein-based recognition systems. The work presented in [1] attempts to more effectively accommodate the potential deformations, rotational and translational changes by encoding the 
orientation preserving features and utilizing a new regionbased matching scheme.

The main features included in palm print are line features, geometry features, point features, texture features and statistical features. In this paper [2] line features are extracted using Complex Gabor Wavelet Transform method. A complex Gabor wavelet is defined as the product of a Gaussian kernel times a complex sinusoid. The line features extracted by complex Gabor wavelet at various values of theta is stored in the feature vector. The feature vector is matched using Hamming Distance similarity measurement using sliding window method.

As a unique biometric feature, palm-print verification has achieved a great success. However, palm-print can alone may not be a solution to meet the high demand of highly accurate and robust biometric systems. Recently, palm-vein, which refers to the palm feature under near-infrared spectrum, has been attracting much research interest in this new era. This paper [7] presents an online personal verification system by fusing palm-print and palm-vein information.

Many kinds of biometric authentication techniques have been developed based on different biometric characteristics. To mention a few are fingerprint, face, iris, palm-print, and hand shape. 2-D palm-print recognition has been widely studied in the past decade and it has been proven that palmprint is a unique biometric identifier. This paper [4] explains the two main approaches to 2-D palm-print recognition: line-based approach and texture-based approach

Palm-print has proved to be one of the most unique and stable biometric characteristics towards the current scenario. Almost all the current palm-print recognition techniques capture the 2-D image of the palm surface. Although 2-D palm-print recognition can achieve high accuracy, the 2-D palm-print images can be counterfeited easily and much 3-D depth information is lost in the imaging process. This paper [8] explores a 3-D palm-print recognition approach by exploiting the 3-D structural information of the palm surface.

Some of the orientation coding based palm-print verification methods, such as competitive code, palm-print orientation code and robust line orientation code, are some of the proven verification algorithms with fast matching speeds. Orientation code makes use of two types of distance measure such as angular distance and OR_XOR. In this paper [5], discusses about a unified distance measure, of which both SUM_XOR and OR_XOR can be regarded as special cases, and provide some principles for determining the parameters of the unified distance for palm-print verification.

The development of accurate and robust palm-print verification algorithms is a critical issue in automatic palmprint authentication systems. Among various palm-print verification approaches, the orientation based coding methods, such as competitive code (CompCode), palm-print orientation code (POC) and robust line orientation code (RLOC), are state-of-the-art ones. In this paper [9], we propose a novel feature extraction algorithm, namely binary orientation co-occurrence vector (BOCV), to represent multiple orientations for a local region.
To improve the accuracy and capability of spoof attacks though 3D images can be addressed to solve the accuracy part, one solution proposed in paper [6] multi-spectral imaging which captures an image in a different variety of spectral bands. Each spectral band highlights specific features of the palm, making it possible to collect more information to improve the accuracy and anti-spoofing capability of palm-print systems.

\section{ADVANCED AND FAST CORRELATION BASED FEATURE FOR PALM-PRINT RECOGNITION (AFCBF)}

A block diagram illustrating the process for palm-print recognition using Advanced and Fast Correlation Based Feature for Palm-print Recognition Filter (AFCBF) is depicted in figure 1.

\subsection{Conceptual Framework of AFCBF}

A typical palm-print recognition consists of five phases. They are (1) Training Images phase (2) Preprocessing phase (3) Feature Extraction phase (4) Database / Search phase and (5) Matcher phase. The palm-print scanner collects the training images of palm-print. Preprocessing involves the setting up of a coordinate system to make significant changes in palm-print images and to segment a part of palmprint image for further processing of feature extraction. Feature extraction includes the process of extraction of effective features obtained from the preprocessed palmprints. A matching process compares two palm-print features and the job of database is to store registered templates

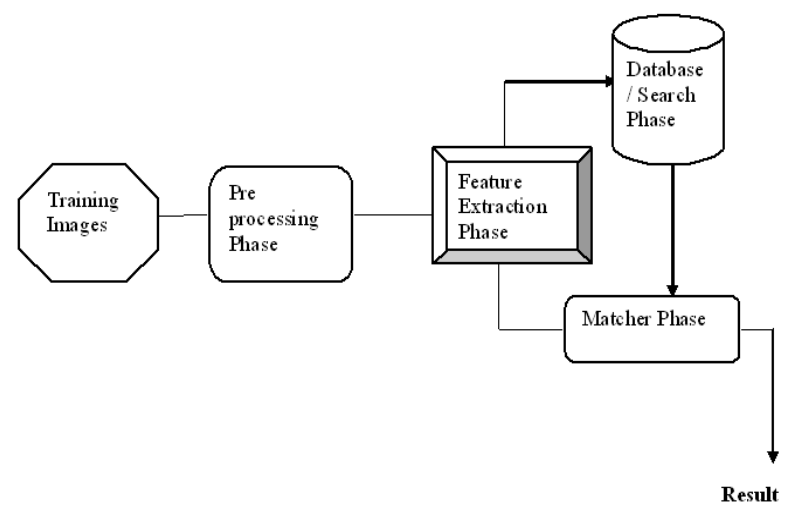

Fig 1 Diagrammatical representation of AFCBF

\subsubsection{Image Training phase}

The first phase involves the collection of training images from the scanner. These scanners make possibility of simplifying the development of recognition algorithms for palm-print because of the images are captured in a controlled environment. In this work we have not used any palm-print scanner for acquisition of palm-print images. Rather we have used the online database of palm-prints using (CASIA Palm-print Image Database) 


\subsubsection{Preprocessing phase}

Before feature extraction phase starts on, it is essential to acquire a sub-image from the captured palm-print image and to discard the different variations occurred due to rotation and translation. From the image obtained, only the central portion of the palm-print image is required for the process of feature extraction. Subsequently the palm-print images have to be properly aligned and the unsuited portions have to be removed away to reduce the computation process required in subsequent processing. The Morphological Texture Segmentation handles the pre-processing tasks, which results in the acquirement of Region of Interest (ROI).

The image preprocessing involves two steps. They are segmentation of palm-print image in a square region and applying morphological operations dilation and erosion from the results obtained after the segmentation. For segmentation a threshold method is applied. The core area is the identification of the best threshold value $k$. The definition is as follows:

$\mathrm{P}_{\mathrm{a}}\left(\mathrm{a}_{\mathrm{b}}\right)=\mathrm{m}_{\mathrm{b}} / \mathrm{m}$ where $\mathrm{b}=0,1,2,3,,, \mathrm{~J}-1$

(eqn 1)

Where $m$ refers to the total number of pixels obtained for palm-print image, $\mathrm{m}_{\mathrm{b}}$ denotes the total pixels having gray level $a_{b}$. From the resultant value obtained two morphological operations dilation and erosion process are applied to remove the noise and ROI are extracted.

\subsubsection{Feature Extraction phase}

The Feature Extraction phase extracts the line features from the palm-print using Advanced and Fast Correlation method which extracts line features from the input palm-print image. The feature extraction portion consists of two steps. In the first step feature ranking is done using spearman rank correlation. The spearman rank correlation for palm print is basically a correlation model defined by the following equation:

$$
\begin{aligned}
& \rho=\frac{\sum_{i}\left(a_{i}-a\right)\left(b_{i}-b\right)}{\sqrt{ } \sum_{i}\left(a_{i}-a\right)^{2} \sum_{i}\left(b_{i}-b\right)^{2}} \\
& \rho=\frac{1-6 \sum j_{i}^{2}}{m\left(m^{2}-1\right)}
\end{aligned}
$$

where $j_{i}^{2}$ denotes the differences $j_{i}=\left(a_{i}-b_{i}\right)$ between the ranks of each observation in the palm print images. The results of the ranking are indexed and stored that result in the ending of the first step. Based on the results obtained in the first step redundant features are removed and unique line features are extracted. Ranking ranges from high to low are compared for the purpose of removal of redundant features based on a comparison algorithm. Lower order ranking consist of redundant features and higher order ranking is considered for extracting of line features.

\subsubsection{Database / Search phase}

The features extracted from the above phase are stored in database as registered templates. This in turn provides palmprint identification in large databases.

\subsubsection{Matcher phase}

To verify the palm-print images Euclidean distances are used. The matching process involves hypothesis generation and evaluation. The hypothesis generation evolves the comparison of two palm-print images by using the following equation 4 and 5 with the help of a predefined threshold value $\mathrm{T}$ for matching of line features in two palmprint templates.

$\operatorname{dist}_{1}=\sqrt{ }\left((\mathrm{a} 1(\mathrm{~m} 1)-\mathrm{a} 1(\mathrm{~m} 2))^{2}+((\mathrm{b} 1)(\mathrm{m} 1)-\mathrm{b} 1(\mathrm{~m} 2))^{2}\right.$

(eqn 4)

$\operatorname{dist}_{2}=\sqrt{ }\left((\mathrm{a} 2(\mathrm{~m} 1)-\mathrm{a} 2(\mathrm{~m} 2))^{2}+((\mathrm{b} 2)(\mathrm{m} 1)-\mathrm{b} 2(\mathrm{~m} 2))^{2}\right.$

(eqn 5)

If dist ${ }_{1}$ and dist ${ }_{2}$ are less than the threshold value $\mathrm{T}$, then it ensures that the two line segments are same. Hypothesis evaluation is performed with the following equation 6 .

$\mathrm{vf}=2 \mathrm{~m} /(\mathrm{m} 1+\mathrm{m} 2)$

where $\mathrm{m}$ denotes the total number of pairs, $\mathrm{m} 1$ and $\mathrm{m} 2$ are line segments of two different palm-print images

\subsection{Proposed algorithm - (AFCBF)}

The proposed algorithm Advanced and Fast Correlation Based Feature for Palm-print Recognition is explained as given below. The following step gives the clear idea of the proposed algorithm AFCBF used for palm print recognition.

Step 1: Acquire training images from the CASIA Palm-print Image Database.

Step 2: Perform preprocessing of palm-print image using segmentation in a square region and applying morphological process.

Step 3: Obtain images from resultant of image preprocessed and perform feature extraction using spearman rank correlation and removal of redundant features using comparison algorithm.

Step 4: Store the template images obtained as a resultant image based on the features extracted.

Step 5: Verify palm-print images using Euclidean distance based on hypothesis generated value and evaluating the same.

\section{EXPERIMENTAL RESULT AND DISCUSSION}

Advanced and Fast Correlation filters classifiers were implemented for palm-print recognition by improving the accuracy for line features. We have analyzed the performance of proposed algorithm AFCBF, using CASIA Palm-print image database. The training sample consists of 5,502 palm-print images captured from 312 users. All palm- 
print images are 8-bit gray level JPEG files by providing the images of palm-print using the database CASIA.

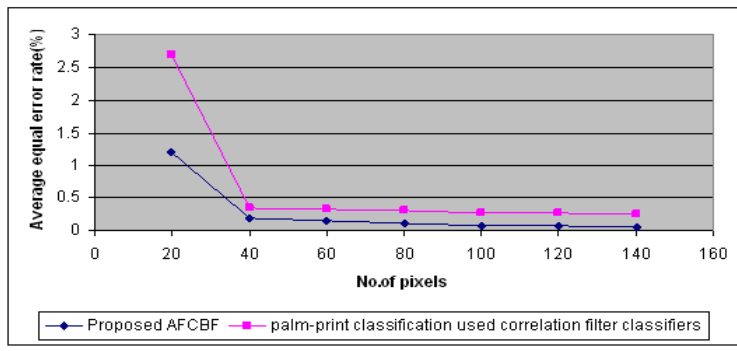

Fig 2: No. of pixels vs. Average Equal Error Rate

Figure 2 illustrates Resultant graph for the comparison of Proposed AFCBF with the existing palm-print classification used correlation filter classifiers. For the experiment, the pixels are taken from $20,40, . ., 140$. The proposed AFCBF is having relatively low average equal error rate when compared with the existing palm-print classification used correlation filter classifiers.

In this experiment, we choose as a region of concern a square in the middle of the palmprint, relative to the two fiducial points of the class.

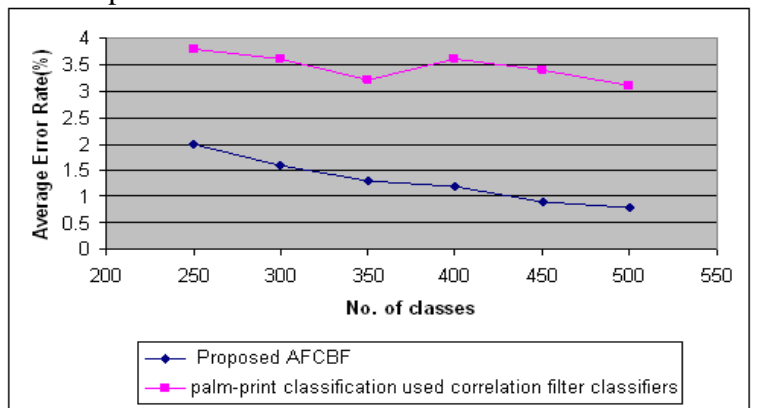

Fig 3: No. of classes vs. Average Equal Error Rate

We show results for the tasks of verification and identification. In verification task, AFCBF address individual thresholds for each class, while we are actually training a set of filters to identify all palm. In verification task, these class or palm-specific thresholds learned from a group of sequestered data in the similar way a global threshold is described. The statistics used for evaluation and comparison in this case is the average equal error rate. The results shown in figure 3 demonstrate our proposed AFCBF method outperforms well.

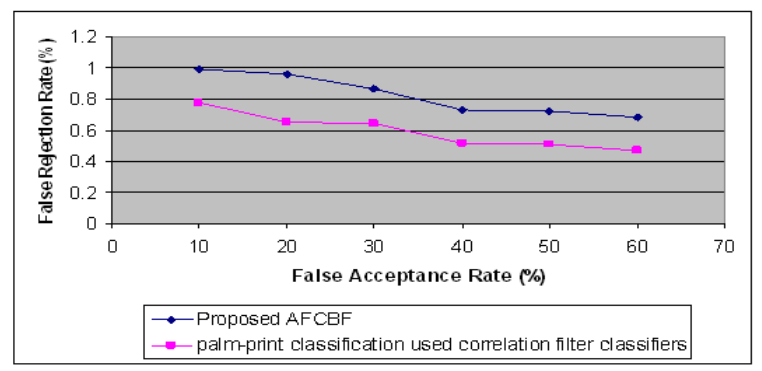

Fig 4: False Acceptance Rate (\%) vs. False Rejection Rate (\%)
Figure 4 shows the comparison results of Proposed AFCBF with the existing palm-print classification used correlation filter classifiers by False Rejection Rate for different values of False Acceptance Rate. The best performance is shown when false acceptance rate low. The results in fig 4 show the best performance of the proposed AFCBF method.

\section{CONCLUSION}

The work presented the scope of analysis of biometric system based on the features of the palm-print by using CASIA database. Although palm-print recognition outperforms using Advanced and Fast Correlation classifier, matching score using hypothesis evaluation improves the system's accuracy.

\section{REFERENCES}

[1] Yingbo Zhou and Ajay Kumar, Senior Member 2011. "Human Identification Using Palm-Vein Images," IEEE Transactions on Information forensics and security, vol. 6, no. 4, December .

[2] Jyoti Malik, Ratna Dahiya, G Sainarayanan, 2011. " Fast Complex Gabor Wavelet Based Palmprint Authentication," International Journal of Image Processing (IJIP), Volume (5) : Issue (3) .

[3] Dr. H. B. Kekre, Dr.Tanuja K. Sarode, Aditya A. Tirodkar," , October 2011. An Evaluation of Palm Print Recognition Techniques using DCT, Haar Transform and DCT Wavelets and their Performance with Fractional Coefficients," International Journal of Computer Applications (0975 - 8887) Volume 32No.1.

[4] W. Li, D. Zhang, L. Zhang, G. Lu and J. Yan, 2010. "3-D Palmprint Recognition with Joint Line and Orientation Features," in IEEE Trans. System, Man and Cybernetics, Part C.

[5] Z. Guo, W. Zuo, L. Zhang and D. Zhang, "A Unified Distance Measurement for Orientation Coding in Palmprint Verification ", Neurocomputing,Volume 73, pp. 944-950, Issues 4-6.

[6] D. Zhang, Z. Guo, G. Lu, L. Zhang and W. Zuo, Feb. 2010. "An Online System of Multispectral Palmprint Verification," IEEE Trans. on Instrument and Measurement, vol. 59, no. 2, pp. 480-490.

[7] D. Zhang, Z. Guo, G. Lu, L. Zhang, Y. Liu and W. Zuo, 3 September 2010. "Online Joint Palmprint and Palmvein Verification," accepted by Expert System with Applications.

[8] D. Zhang, G. Lu, W. Li, L. Zhang and N. Luo, Sept. 2009. "Palmprint Recognition using 3-D Information," IEEE Trans. System, Man and Cybernetics, Part C, vol. 39, no. 5, pp. 505-519.

[9] Z. Guo, D. Zhang, L. Zhang and W. Zuo, OCT 2009."Palmprint Verification using Binary Orientation Co-occurrence Vector," Pattern Recognition Letters,vol. 30, no. 13, pp. 1219-1227. 